\title{
Estandarización del Modelo de Negocios para el Modelado Estándar de Datos
}

\section{Standarization of Business Model for Standard Data Modeling}

\author{
Tino Eduardo Reyna Monteverde ${ }^{1 *}$ \\ ${ }^{1}$ Facultad de Ingeniería Industrial y de Sistemas de la Universidad Nacional de Ingeniería, Lima Perú.
}

\begin{abstract}
RESUMEN
Este artículo presenta un nuevo enfoque para determinar El Modelo Funcional de Negocio que se requiere para el modelo de datos del futuro sistema automatizado. El alcance de este nuevo esquema cubre la típica actividad empresarial que requiere de sistemas de información para la toma de decisiones empresariales. Se analizan los patrones de las áreas funcionales de toda empresa, llegando a presentar los esquemas estándar para cada una de ellas. Estos patrones son demostrados y sustentados debidamente. Los Sistemas Informáticos durante su vida útil sufren una serie de ajustes vía mantenimiento. Estos cambios generan muchos costos en tiempo y dinero. La estratégia para evitar esta situación es tomar como base un modelo de negocio estándar, el cual pueda ser personalizado vía parâmetros de acuerdo a los requerimentos particulares del usuário. Con el objetivo de encontrar el Modelo estándar de negocio se demuestra que son cuatro las Macro funciones básicas que de manera universal deben estar presentes en toda área de Negocios. Estas macro funciones son presentadas y detalladas. Para llegar a este esquema se demuestra que el servicio entregado por todas las áreas que forman la empresa son Productos tipo Servicio y por lo tanto deben cumplir de manera holística con el Ciclo de Vida de Producto (cuatro etapas del Ciclo). Los modelos se presentan tanto de manera gráfica como analítica. En base a este modelo de negocio estándar, se determinarán los requerimientos de información, imprescindibles para la etapa de análisis de los Sistemas de Información.
\end{abstract}

Palabras clave.- Etapa estudio del sistema, Análisis de sistemas, Requerimientos funcionales, Modelo de negocios, Diagrama funcional.

\begin{abstract}
This paper presents a new approach for determining the functional business model required for the data model of the future automated system. The scope of this new scheme covers the typical business that requires information systems for business decision making. Patterns of functional areas of any enterprise are analyzed, reaching present standard schemes for each. These patterns are displayed and properly supported. Information Systems for life undergo a series of adjustments via maintenance. These changes generate many costs in time and money. The strategy to avoid this situation is to build on standard business models, which can be customized according to parameters in particular. In order to find the standard business model there are four basic functions Macro universally must be present in all areas of business. These macro functions are presented and detailed. To reach this scheme shows that the service provided by all areas that form the company are type Service Products and therefore must comply holistically with the product life-cycle management (four stages of the cycle). The models are presented both graphically and analytically. They are determined iOn requirements, essential for the analysis stage of the Information Systems based on this standard model business
\end{abstract}

Key words.- Stage study system, System analysis, Functional requirements, Business model, Functional diagram.

\section{INTRODUCCIÓN}

\section{IMPORTANCIA DEL MODELO FUNCIONAL DE NEGOCIOS}

Uno de los problemas fundamentales para lograr plantear la Arquitectura del Modelo de Datos para un Sistema de Información, radica en determinar los requerimientos funcionales del área de negocios [1], porque al determinar estas funcionalidades, se podrá definir el marco de especificaciones mínimas a ser consideradas en la Arquitectura de Datos, asegurando la Robustez Semántica deseada, certificando un nivel mínimo de mantenimiento futuro y estará listo para la evolución del sistema en su adadptación hacia nuevas tecnologías [1] (Modelo Estable).

En el equema tradicional se advierte las siguentes desventajas [05]:

\footnotetext{
La Revista Científica TECNIA protege los derechos de autor bajo la Licencia 4.0 de Creative Commons: Attribution 4.0 International (CC BY 4.0).

* Correspondencia:

E- mail: treyna@uni.edu.pe
} 
1. Se basa en una mirada de una comunidad de usuarios que comparten la misma cultura organizacional.

2. Generalmente precisan necesidades para el corto plazo.

3. Tanto las Reglas como las Políticas de Negocio, cambian o evolucionan, sin embargo en la mayoría de los casos estas se encuentran embebidas en el código de los programas manteniéndoce rígidas sin la capacidad de ser modificadas o adecuadas a nuevos entornos.

De acuerdo con Boehm [2] "relative to removing a defect discovered during the requirements stage, removing the same defect costs on average 3.5 times more during design, 50 times more at the implementation stage, and 170 times more after delivery".

\section{Situación actual}

Se han venido haciendo muchos esfuerzos para lograr determinar la mejor manera de especificar los requerimientos funcionales. Entre las mas conocidas se encuentra el diagrama de Casos de Usos (UML[6]), Ingeniería de Requerimientos, BPM, RUP entre otros. Sin embargo, se siguen basando en el modelo tradicional es decir, en lo que desea el usuario; con lo cual se obtiene un esquema personalizado cuya validez es relativa para el momento en que se realizó el estudio y a un grupo humano que la definió limitando su Robustez Semántica.

Argumentación.- Al plantear los usuario sus requerimientos lo hace desde su punto de vista y sus propias necesidades, que para el momento en que lo solicita puede parecer correcto. Probablemente para otros usuarios puede ser insuficiente, incluso para los mismos usuarios tiempo después les parecerá limitado e incompleto. Se debe pensar en la estabilidad del modelo la misma que asegure robustez para el presente y futuro. Se han hecho algunos avances para determinar el Modelo Funcional de Negocio, pero no se ha encontrado la metodología para ello, ni el paradigma que lo sustente [15]. Apelando inclusive a la Tormenta de Ideas[1]. El tradicional esquema funcional, el cual presenta el diagrama en forma de organigrama, tiene la estrategía de ir descomponiendo las macros funciones en funciones simples [4]. Sin embargo no es una guía ni asegura que se hayan considerado el Modelo Funcional de Negocio de manera completa para mantenerse en el tiempo.
Argumentación.- La técnica de la descomposicón funcional no es estricta además las funciones tienen categoría de macro-funciones, funciones, mini funciones sin tener claro cuando se debe terminar de descoponer. Permite una libertad que es mal utilizada que no tiene control ni guía del desarrollo. Cada quien la utiliza aplicando su criterio o sentido común, generado esquemas personales y no consensuados. En este artículo se presenta un método riguroso desde el punto de vista semántico [7] que constituye una guía y asegura una adecuada Robustez Funcional, apelando al Paradigma "Orientado al Negocio" (Enfoque que permite conceptualizar al Área de Negocio como generadora de un Producto tipo Servicio), el cual brinda un nuevo enfoque a la manera de plantear el Modelo Funcional de Negocios (Creando Sinergia al juntar elementos del Negocio).

\section{MODELO FUNCIONAL DEL NEGOCIO}

El objetivo de la etapa de estudio de un proyecto informático, es determinar las funciones del área que se desea automatizar (Modelo Funcional de Negocio). Este Modelo Funcional es el conjunto de Funciones que hacen posible el propósito del Área Orgánica de Negocio [4].

Considerando:

Función = Actividad propia de un Ente

Actividad $=\{$ Actos o Eventos $\}$

\section{REALIDAD}

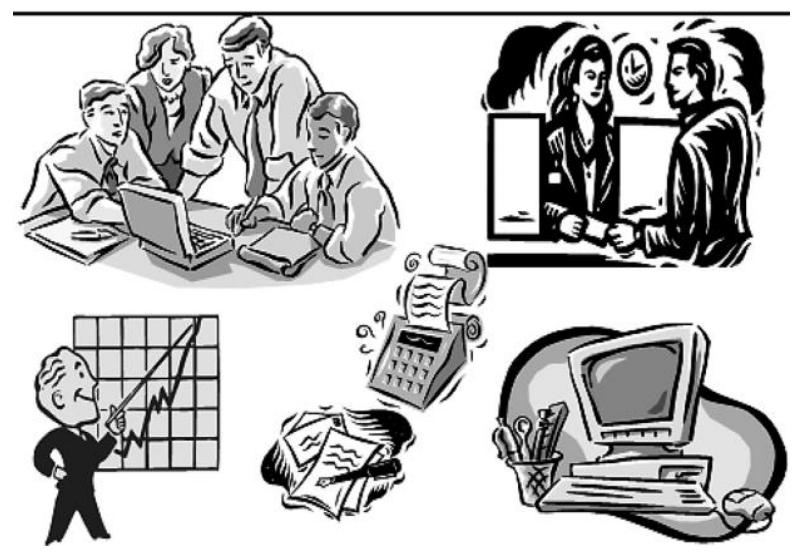

Figura 1. La realidad es compleja, confusa y ambigua.

Luego:

Función $=\{$ Eventos $\}$ propios de un Ente

Etapa: Estudio del sistema 
EMPRESA COMO UN SISTEMA.

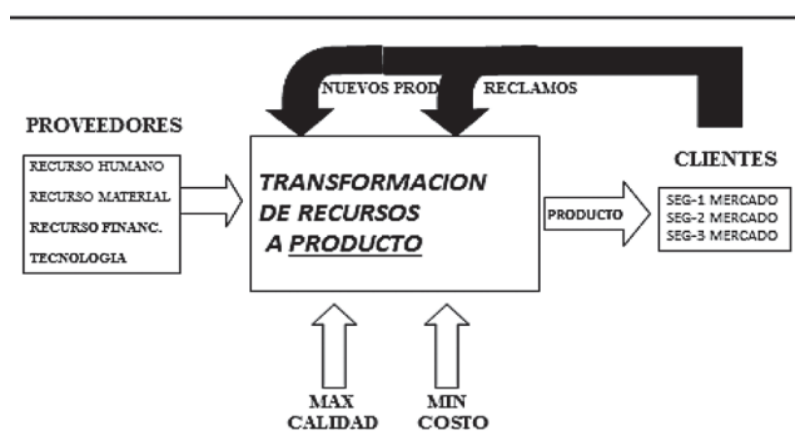

Figura 2. Esquema conceptual de todo negocio.

EMPRESA COMO UN SISTEMA

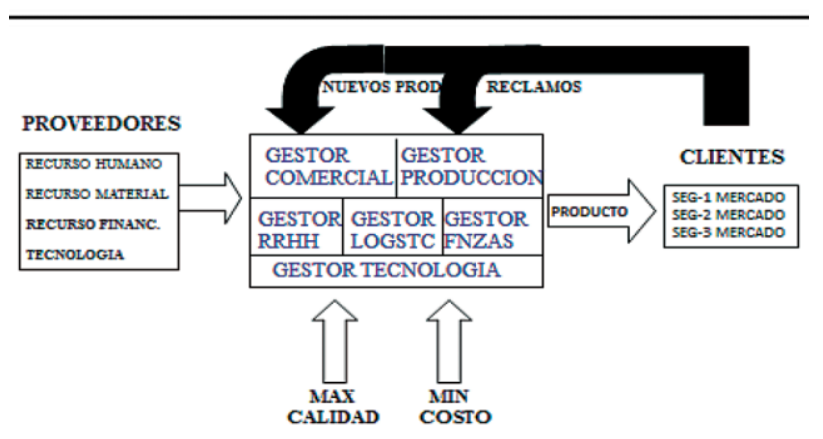

Figura 3.Abriendo la caja negra, detallando funciones.

Cuando se inicia esta etapa se pueden tomar varias fuentes para conocer el negocio, sin embargo la más típica (tradicional) es el acercarse al área misma del negocio, observarlo y extraer de él el conocimiento requerido. Tomando al usuario como única fuente. $\mathrm{Al}$ enfrentarse a la realidad se encuentra que ésta no es capaz de entregar de manera directa y clara sus propiedades y características porque la realidad es:

- Compleja (compuesta por muchas variables)

- Ambigua: Se percibe o interpreta (Cada usuario la enfoca de acuerdo a su necesidad y experiencia, interpretación filosófica de Kant)

- Muchas veces no está formalizada. Figura 1.

Se debe precisar que el alcance de la presente investigación es a nivel empresarial [14], luego el estudio de esta realidad se limitará a ella. A continuación se presenta el esquema general de toda organización empresarial que genera Negocio.

\section{Esquema general de negocio}

Toda empresa al estar compuesta por un conjunto de elementos (personas, cosas, procedimientos, etc.) y con un objetivo definido, es conceptuada como un sistema [18]. De acuerdo a la Figura 2, en esta se observa de manera muy nítida los componentes de todo sistema vale decir:

- Entradas

- Procesos

- Salida

- Parámetros y Retro-alimentación.

Es importante aplicar el enfoque de sistema hacia 
el negocio porque nos informa de manera holística su naturaleza, bajo esta perspectiva la empresa es un gran proceso transformador de recursos en productos bajo el control de los parámetros de Calidad y Costo. La retro-alimentación informa sobre las mejoras o suplementos necesarios a los productos. Ampliando la visión de este gran módulo se detallan los componentes que siempre están presentes en toda empresa como [16]:

- Función Comercial

- Función Producción

- Función RRHH

- Función Logística

\section{- Función Financiera}

- Función Tecnológica

Estas funciones están presentes en toda organización en diferentes magnitudes y formas, sean automatizadas, manuales o semi-automatizadas, desde la gran empresa, mediana, pequeña incluso microempresa. La diferencia fundamental entre estas es el volumen de producción.

\section{Detalle funcional de negocio}

Sustento:

Los clientes en sus diversos segmentos del mercado,

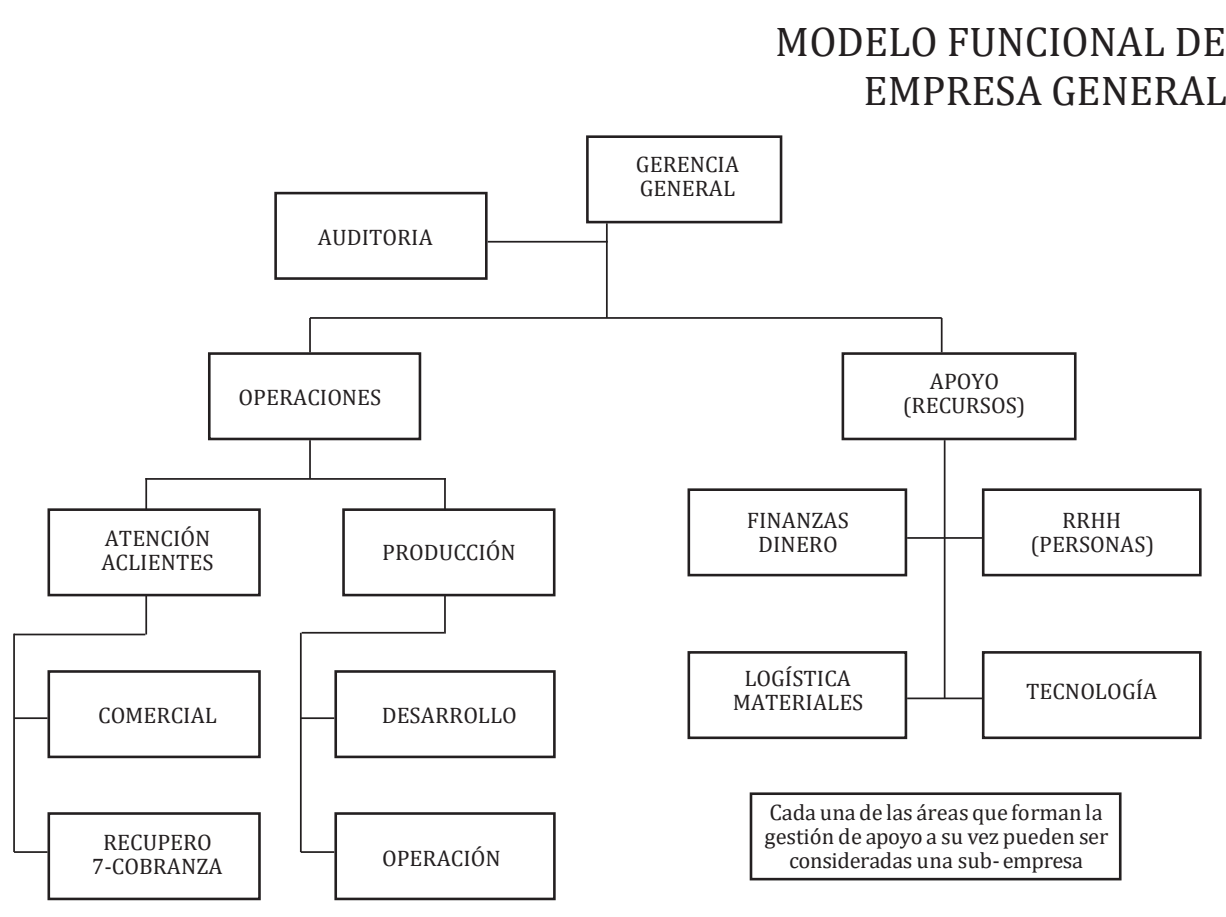

Figura 4. Organización universal en toda empresa.

interactúan con los gestores de la empresa como: Ver Figura 3

- Comercial y

- Producción

Ya sea demandando nuevos productos o por mejoras en los actuales. Así estas dos grandes macro funciones constituyen el corazón del negocio, las mismas que a su vez deben ser apoyadas por un conjunto de recursos como:

- Humanos,
- Materiales,

- Financieros y

- Tecnológico.

Este grupo de macro-funciones desarrollan el rol de apoyo al núcleo.

Considerando que el servicio es un ente intangible, compuesto por operaciones que no generan bienes (según acepción del Diccionario de la RAE) ejemplo: Transporte, Salud, Educación, etc. 


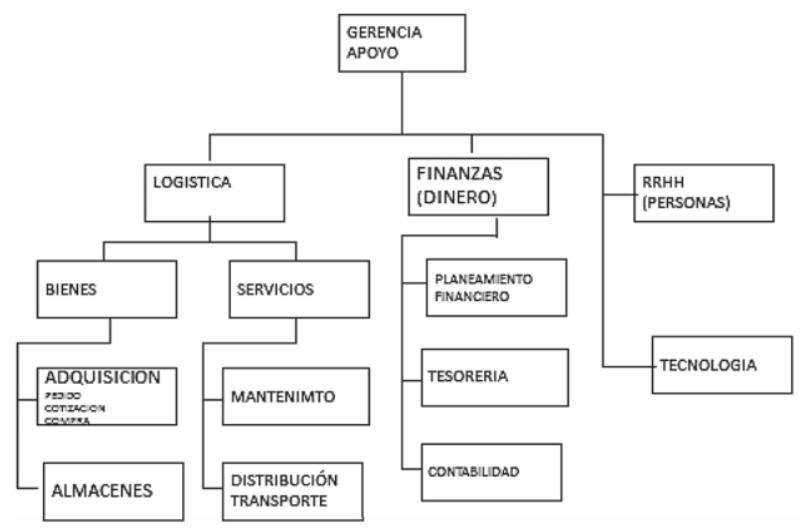

Figura 5. Las áreas de apoyo dan soporte a las operativas.

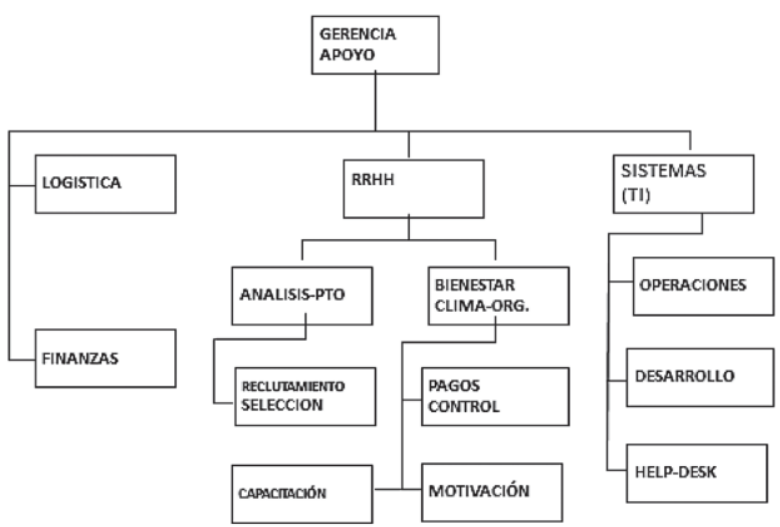

Figura 6. Organización típica de sistemas administrativos.

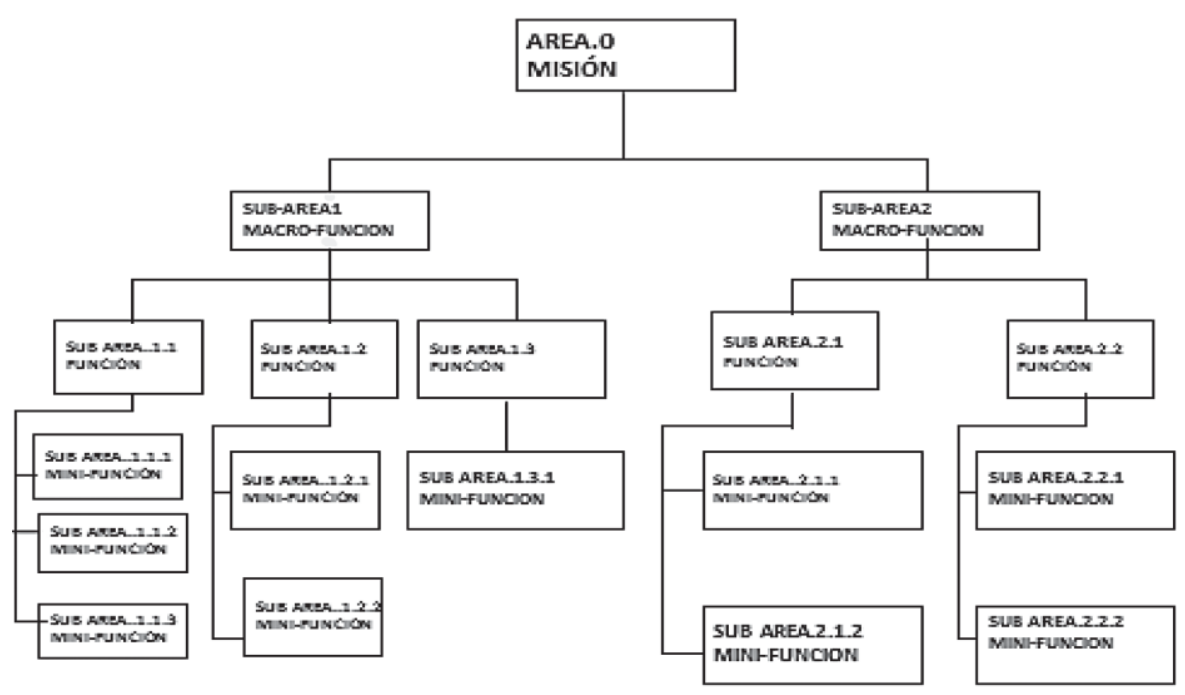

Figura 7. El área de compras detalla en un conjunto de funciones especializadas. 
Luego:

Sí Área de Negocio = Función

\& Función $=$ Servicio

\& $\quad$ Servicio $=$ Producto

Se concluye:

$$
\text { Área de Negocio }=\text { Genera (Producto) }
$$

Esta conclusión, presenta a las áreas funcionales como generadoras de productos. Por otro lado los modelos funcionales describen como funcionan las áreas de la empresa. Estos modelos se presentan bajo un esquema de jerarquías de funciones porque estas se van descomponiendo en otras más simples.

Toda empresa u organización se debe entender como un conjunto de áreas donde cada una realiza una función especializada $\mathrm{y}$, entregan un producto (servicio) a la empresa. Esta conclusión cambia el paradigma del sistema empresarial. Las funciones de cada área son de naturaleza universal y constante sin importar el giro de negocio, nacionalidad ni envergadura.

El carácter universal se comprueba con la globalización, que homogeniza la forma de hacer negocios en el mundo. La importancia del fenómeno de la globalización radica en que sistemas de cualquier parte del mundo funcionan esencialmente iguales, permitiendo implantar modelos desarrollados en lugares lejanos, siendo personalizados mediante el ajuste de parámetros.

\section{Modelo funcional del negocio}

Todas las funciones de las empresas se agrupan en dos grandes bloques las: Operativas y las de Apoyo, Figura 4.

Las primeras están orientadas hacia el núcleo (razón de ser) del negocio y las de apoyo dan soporte a las primeras. De allí que muchas veces estas funciones son realizadas por terceras empresas.

Todas estas áreas se organizan para conseguir la gran misión o razón de ser de la empresa. A continuación se detallan estos bloques.

Áreas de Apoyo (Gestión Administrativa), Figura 5 y 6. [13].

A. Logística.- Su razón de ser es la de proveer bienes y servicios a todas las áreas de la empresa. Sobre todo a las áreas operativas que son la razón de ser de toda organización. Debe velar por el mantenimiento en estado óptimo de todo recurso físico del negocio.

B. Recursos humanos.- Su razón de ser es la proveer personal capacitado de acuerdo a un perfil diseñado según el puesto de trabajo al cual será asignado. Debe velar por el óptimo desempeño del personal.

C. Finanzas.- Su razón de ser en la empresa es la de proveer los fondos necesarios para el normal funcionamiento. En los casos de excedentes colocara a la mejor tasa del mercado y en el lugar más seguro. En los casos de déficit deberá financiarlo mediante préstamos a entidades externas financieras o no en las mejores condiciones para la empresa.

D. Tecnología.- Su razón de ser es la de brindar el soporte tecnológico tanto en los proyectos de automatización así como a los sistemas en la fase de producción. Estas áreas descritas brevemente en su propósito, son de naturaleza transversal al interactuar con todas las demás áreas. Se puede considerar una infraestructura de segundo nivel. Tienen por misión

CATALOGO DE PRODUCTOS (TIPO SERVICIO)

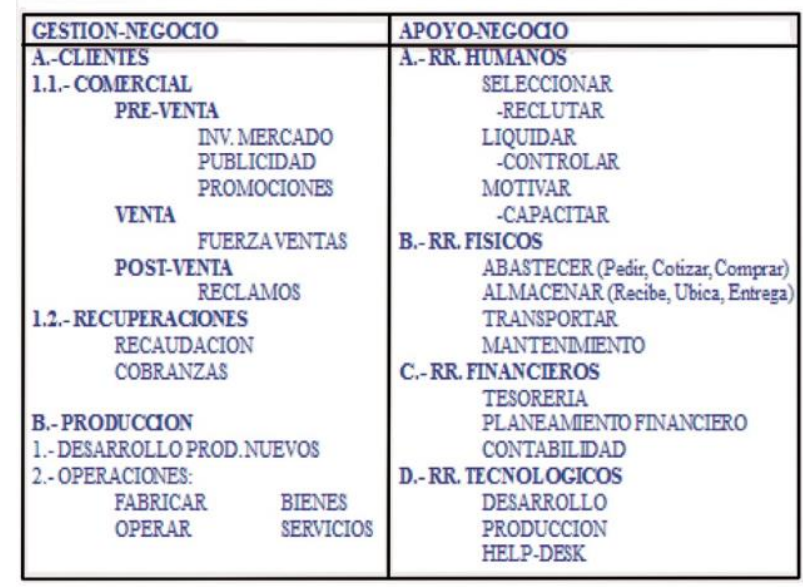

Figura 8. La empresa vista como un catálogo de productos.

apoyar a las del Núcleo del Negocio, proveyendo de recursos como bienes y servicios, para asegurar la continuidad operativa de la empresa.

\section{Áreas del Núcleo del Negocio}

Estas áreas están conformadas por un conjunto de funciones que determinan el propósito del negocio. Estas se concentran en el área de Operaciones de la empresa, la cual a su vez se divide en los Módulos:

A. Clientes 


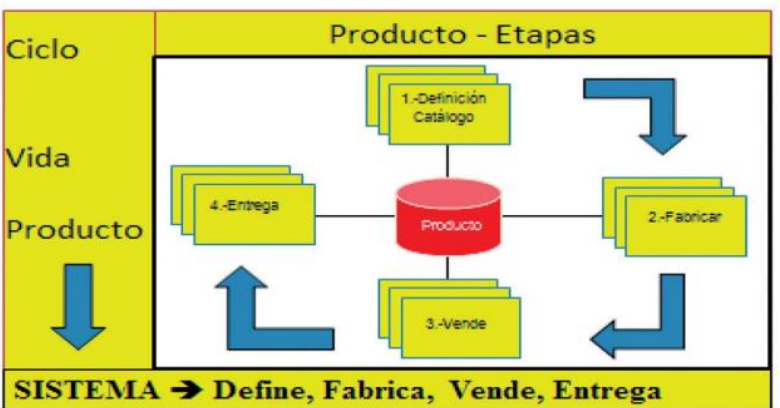

Figura 9. Ciclos de vida de productos.

1. Comercial
a. Pre-Venta (Mercadeo y Promoción)
b. Fuerza Venta (Fuerza deVentas)
c. Post-Venta (Reclamos y Soporte)

2. Recuperación
a. Cobranza

B. Producción

1.- Desarrollo de Productos

(Innovación, Creación Productos)

2.- Operación

(Producción y Facturación)

Luego:

\section{Modelo Negocio $=($ Funciones $/$ logran propósito Negocio)}

Utilizando el simbolismo matemático se lee como:

Modelo de Negocio es igual a un conjunto de Funciones tales que logran el propósito del Negocio. Esta es una definición sintetizada y, amplia a la vez, porque su alcance cubre las funciones actuales y las que posiblemente puedan emerger en el futuro. Sin embargo, hace falta encontrar la estructura de estas funciones, pues no basta con conocerlas sino además se debe precisar cómo se relacionan entre ellas, a
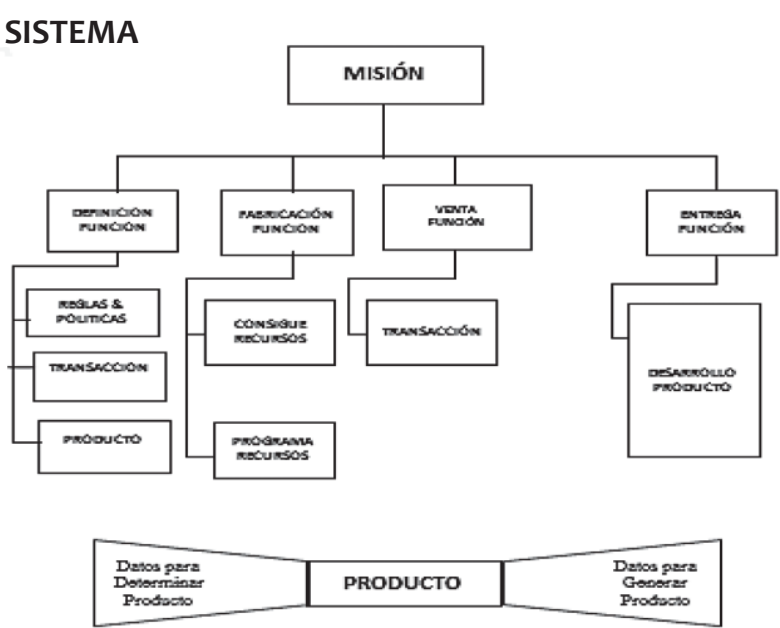

Figura 10. Macro-Funciones de toda área funcional. nivel de detalle y complementación, Figura 7. Estas funciones se complementan unas a otras, de manera que en conjunto resuelven el trabajo de la empresa.

Las funciones deben ser ejecutadas para que se materialicen. Toda ejecución se da mediante procesos los cuales se inician con el nacimiento de un evento [10]. Todo evento genera cambios, en las entidades que participan en el mismo, siendo estas entidades impactadas o modificadas en los valores de sus atributos.

Con la Re-Ingeniería y Mejora Continua de Procesos, estos se encuentran en constante cambio

\section{1-NATURALEZA DE PRODUCTO}

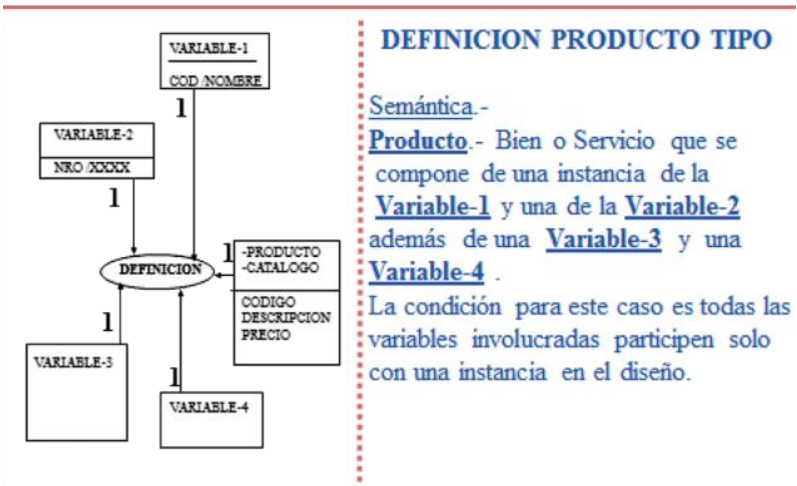

Figura 11. Esquema de la estructura de un producto en función de sus componentes.

mejorándolos o siendo reemplazados por otros de mejor performance [11]. De allí su inestabilidad de los procesos en el modelado de estos por lo que no amerita considerarlo en esta etapa. Por lo expuesto en el párrafo anterior, la propuesta de este artículo es no considerar a los procesos en la etapa de Modelado del Negocio, colocando el foco de atención en las Misión, Funciones, Eventos y Cambios de valores de Atributos.

Los cambios asimilados por los datos, se evidencian en sus estadísticas mediante las cuales se describe el comportamiento de las entidades (totales netos de los impactos de eventos del sistema). Todo cambio al sistema se evidencia al comparar el estado de los datos de los objetos.

Estos estados pueden ser: creados, eliminados o alterados en su valor inicial (modificado) generando un "Parque de Datos de Objetos" (conjunto de datos generados por la acción de eventos, hechos a partir 
de sus Clases).

\section{Núcleo del negocio}

Por ser la razón de ser del Negocio la Producción y Venta del Producto luego estas macro-funciones se constituye en el Núcleo del Negocio.

\section{Producto}

Se entiende por Producto, todo aquello que sea susceptible de ser tranzado: vendido, comprado, devuelto, etc. Es el objeto de intercambio que participa en toda transacción, donde se establece un acuerdo entre el Negocio y el Cliente. En todo evento (Transacción ejecutada) el producto adquiere el rol de existencia (producto fabricado, listo para la venta) el cual puede ser un bien (tangible) o servicio (intangible). Nos centraremos en el modelado de productos tipo servicio.

Por naturaleza de todo producto está compuesto por un conjunto de elementos que se sintetizan en un ente sinérgico único con personalidad propia. Ejemplo el Servicio de Vuelo comercial se compone de Lugar Origen, Destino, Tipo de Nave, Horario y Categoría de asiento. Los elementos que conforman la estructura de todo producto, se agrupan en Técnicos y Comerciales. Los elementos técnicos dependen de la

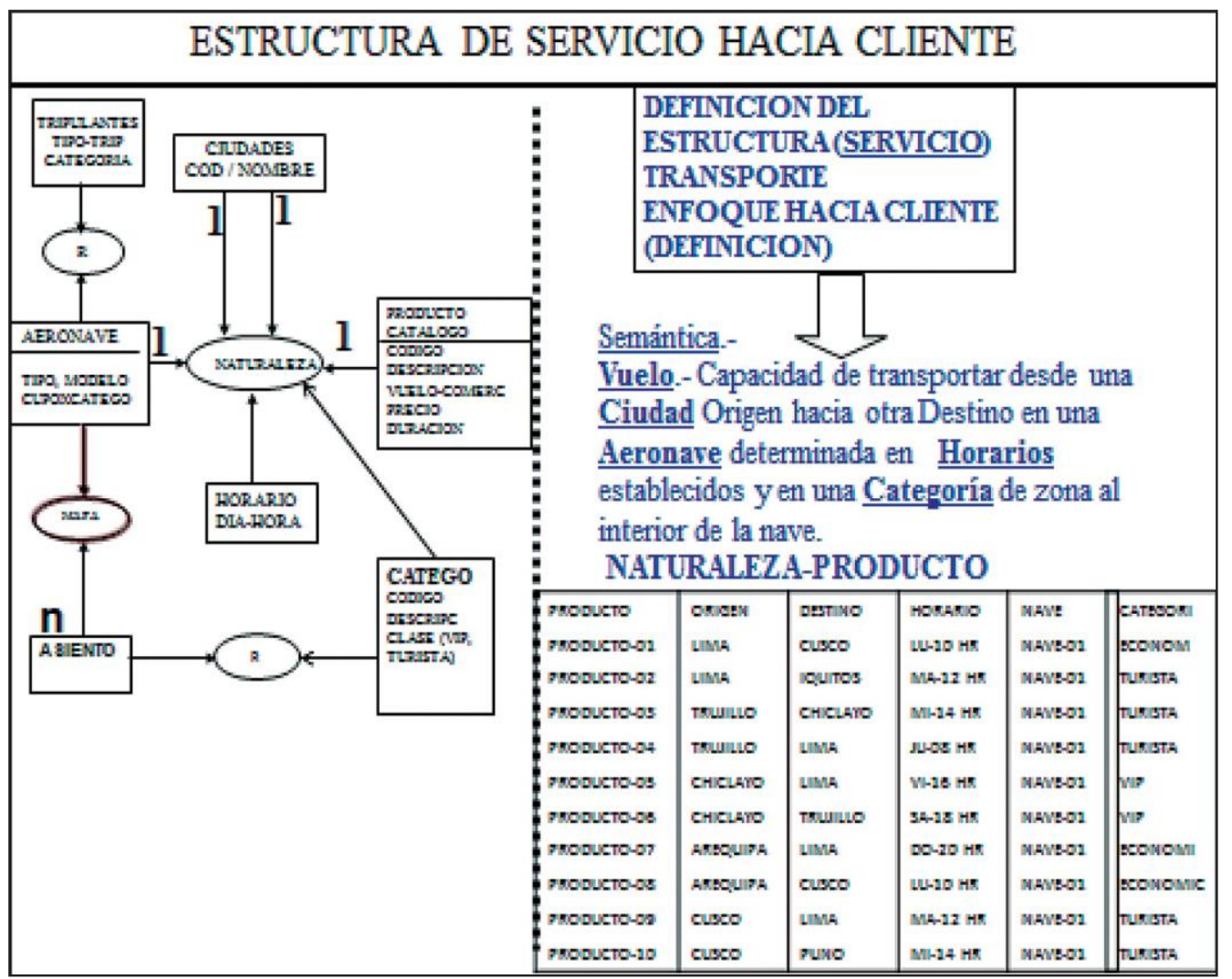

Figura 12. Para el producto Vuelo Aéreo se muestra los componentes de su naturaleza.

naturaleza intrínseca del producto (servicio), para el ejemplo del vuelo los elementos son lugar de origen, destino y tipo de nave. Los elementos comerciales dependen de las condiciones del mercado, Como son horario y categoría. Tanto los elementos técnicos como los comerciales influyen en su atributo Precio (del servicio).

\section{Catálogo de productos}

Como cada área funcional se comporta como una pequeña empresa que brinda un servicio (producto) a la empresa en su conjunto, en esta se generan un catálogo de productos. Estos productos se dan tanto en el área del Núcleo como en las áreas de apoyo a esta. Al convertirse las áreas en generadora de productos, toda empresa genera un gran catálogo de estos, Ver Figura 8.

Como todo producto los servicios deben cumplir con su Ciclo de Vida Universal que consiste en las siguientes etapas: Definición, Fabricación, Venta y Entrega, Figura 9. Estos cuatro ciclos deben estar presentes en la operativa normal de toda organización. 
Estos ciclos se detallan desde el enfoque funcional como uno de los aportes del artículo. Este aporte consiste en presentar la estructura y relaciones que deben existir entre estas funciones.

\section{Ciclo de vida del producto}

1. Definición de Producto.- Su Naturaleza (componentes), Transacciones, Reglas y Políticas de Negocio.

2. Fabricación de Existencias.- Determinar recursos y fecha de entrega programada.

3. Ventade Productos.-Asignar el Producto Fabricado (programado) al Cliente.

4. Entrega de Productos.- Asegurar Calidad en la entrega a través de Protocolos de Operación.

Así toda área funcional de la empresa deberá desarrollar como mínimo estas cuatro etapas a fin de cumplir con su objetivo de entregar su producto a las áreas que se lo demanden. El impacto sobre los organigramas funcionales de toda empresa será que todas deberán tener estas cuatro Macro-Funciones como parte de toda Área Funcional.

\section{Funciones del ciclo vida del producto}

Se detalla cada uno de las etapas de del ciclo de vida, Figura 10.

\section{Definición de productos}

En este ciclo se precisan las características y propiedades de los productos, antes de ser fabricado. Esta función determina la naturaleza del producto, detalla los componentes y las relaciones entre ellos. Todo producto resulta de la composición de " $n$ " elementos, los cuales son independientes entre sí, pero cuando se define el producto se crea una relación entre ellos determinando la estructura del producto, como se aprecia en la Figura 11. Dando origen a un ente con personalidad propia, no la simple mezcla de elementos. Se consigue la Síntesis.

La característica fundamental de estos componentes es que las instancias u objetos de cada componente pueden ser seleccionados por los mismos clientes (vía Combo box).

El Precio es un atributo del producto y está en función de la calidad de las instancias seleccionadas. De hecho existe una relación directa entre calidad o categoría de instancias seleccionadas por componente y Precio Final hacia el cliente. Los atributos del producto

\section{GENERACION DE TICKET DE VUELOS}

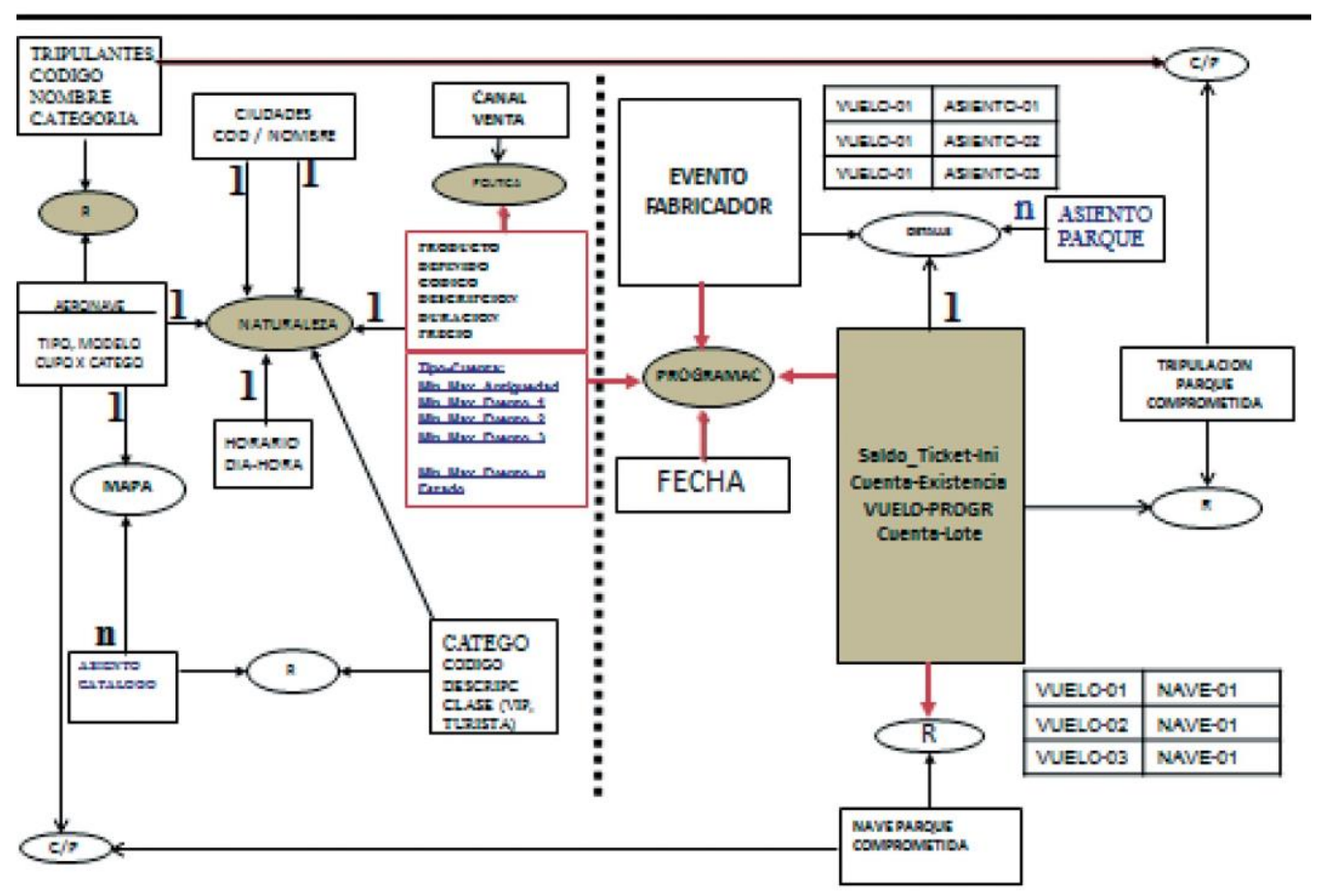

Figura 13. Se observa un lote de tickets generados. 
además del precio son pocos, porque el detalle de características está dado por los elementos que lo conforman.

Se genera una combinatoria entre las instancias de los elementos por la variedad de valores que pueden tomar cada componente del producto. Es así que el producto genera su propio catálogo conteniendo el conjunto de combinatorias posibles de los valores de cada componente. Tema clave en esta macro función es, "descubrir" la naturaleza del Producto. Estructura de la combinatoria de los componentes. En la siguiente Figura 12, se presenta un ejemplo de naturaleza de un Producto. Conocido como Vuelo Comercial el cual se compone de los siguientes elementos: (modelo simplificado).

\section{Componentes técnicos:}

- Ciudad: Origen, Destino -> Catálogo Ciudades

- Tipo Nave -> Catálogo de Modelos de Naves Componentes Comerciales:

- Horario -> Se consideran 7 x 24

- Categoría -> Económica, Turista, VIP

Esta definición del producto establece un precio. Este atributo del producto es la síntesis de esta combinatoria. Es una variable dependiente de " $n$ " factores.

Para este ejemplo:

\section{Precio = F (Origen, Destino, Tipo-nave, Horario, Categoría).}

Al igual que el atributo Precio, los otros como duración, distancia, etc. igualmente tendrán la misma dependencia. La técnica para determinar si una entidad es o no parte del Producto es si al variar una instancia de ella varía el valor de su Precio (Sensible al precio). En el ejemplo se observa la generación de un catálogo con las combinatorias de los valores de las entidades que componen el Producto.

El producto cartesiano de estas entidades genera el catálogo completo de productos (Clase Producto). A partir de cada instancia del catálogo se podrán fabricar " $n$ " objetos (tickets de vuelo) stock de Existencias de cupos. Sobre la base de la Naturaleza del Producto (Núcleo del Negocio formado por las entidades relacionadas directamente con el Producto) se complementa el modelo con entidades propias del negocio en un segundo nivel (para el ejemplo de Vuelo, Tripulación y Asientos). Sobre este esquemase adicionarán componentes como Reglas y Políticas de Negocio.

Las reglas sobre el producto.- Pueden hacer variar el valor de algún atributo por ejemplo el Precio de Catálogo (descuentos por ofertas).

Las Políticas con el Producto.- Determinan la factibilidad de alguna Operación de Negocio, se dé o no (ejemplo: permite descuento o no).

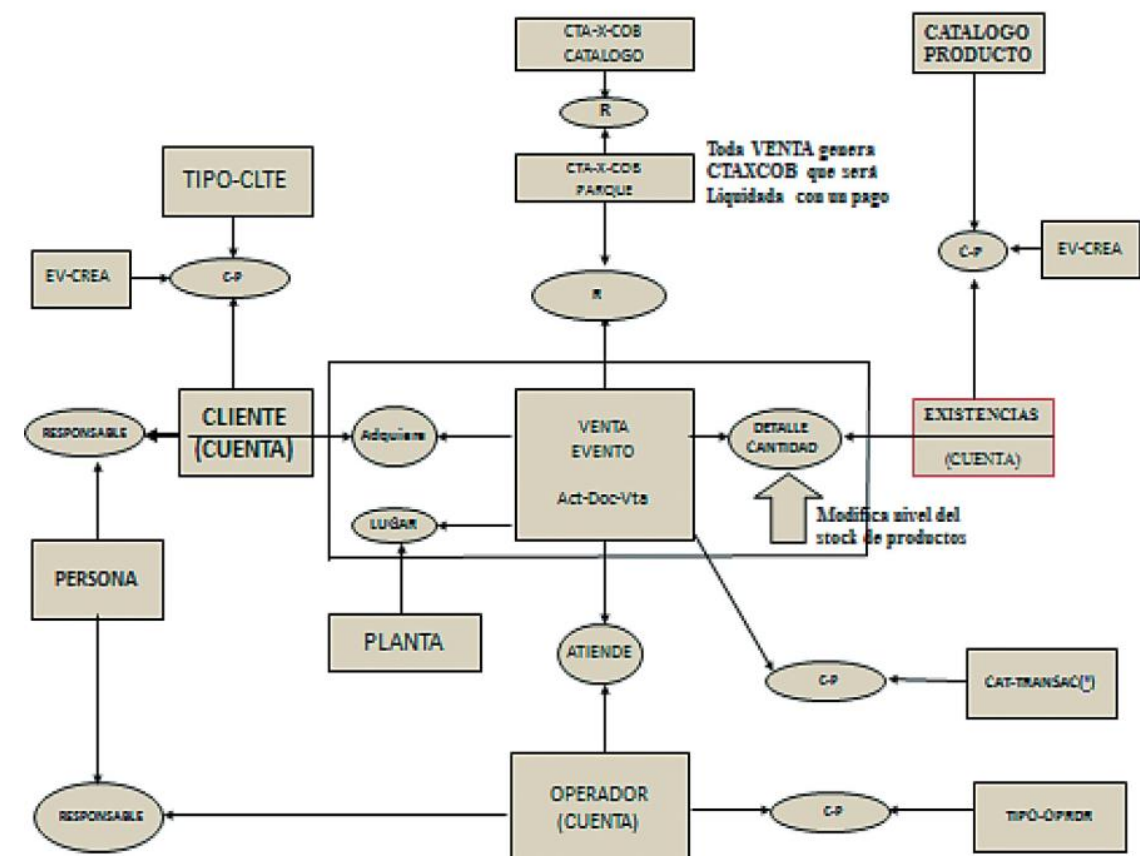

Figura 14.Transacción de venta y sus componentes. 
Además todo Producto debe definir el Protocolo para fabricación, venta y entrega.

\section{Fabricación de productos}

Este enfoque considera al producto fabricado cuando se pueda realizar la Venta de este (no significa entrega inmediata). Un Producto tipo Servicio realmente se produce (Fabrica) cuando se Entrega. Un Servicio se considera Fabricado cuando se tiene la certeza en Entregarlo al cliente en un Eventofuturo.

\section{Servicio Fabricado <> Listo para la Venta}

Este enfoque permite poner atención a todas las actividades y recursos que deben quedar listos antes de su venta (no significa entrega). En el tema de servicios, se deben consideran todos los elementos necesarios disponibles para la entrega del servicio de manera coordinada. Esto implica fijar la concurrencia de estos elementos en fechas ofertadas a los clientes. El concepto de Ticket resume esta característica del servicio. Todo Ticket indica la existencia de un evento futuro, en el cual un conjunto de elementos necesarios para la entrega del servicio están comprometidos. Al darse el compromiso del servicio vía ticket significa que existe una disponibilidad finita equivalente a un límite de cupos, reservas, turnos, etc. Está implícito la limitación de recursos sean estos materiales, financieros, tecnológicos o humanos, Figura 13.

Por ejemplo para el servicio médico en alguna clínica cada cita requiere la disponibilidad de un médico y un consultorio (salvo sea a domicilio). Para productos más sofisticados como los Préstamos Bancarios se requerirían de fondos monetarios además de operadores.

Luego los tickets no significan en sí el Producto, sino la promesa que el servicio será brindado (en la fecha, hora y lugar pactado) lo cual lo hace factible de ser comercializado como cualquier bien tangible (stock disponible).

En todo evento futuro (ticket) se debe precisar:

1. Servicio Programado (Lote de Tickets)

2. Fecha y hora Programada (según tipología)

3. Código del Servicio (naturaleza del servicio)

4. Evento que generó el lote detickets

5. Cuenta Control de Existencias de Tickets

6. Operador programado para entregar servicio

7. Planta o lugar de entrega del servicio.

Cuando se realiza un requerimiento a través de: solicitud, pedido, venta, reserva, matrícula etc. el sistema como respuesta le asigna un ticket a este requerimiento. Porque los ticket fueron generados anticipadamente antes de ser demandados. Este ticket es la materialización de un derecho a recibir el servicio correspondiente en un evento futuro detallado en él mismo ticket.

La entidad fecha.- Es la responsable de realizar el sincronismo entre los elementos necesarios para la

\section{Desarrollo de Transacción Entrega}

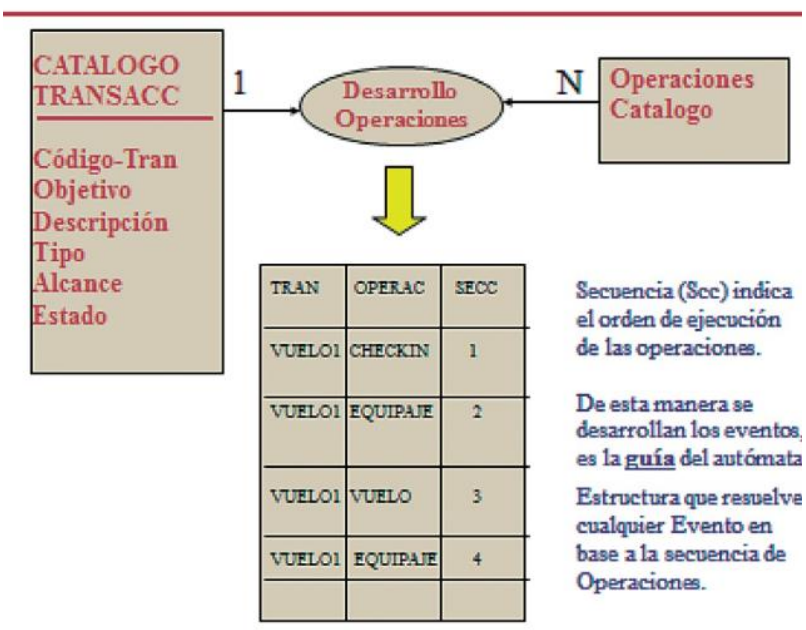

Figura 15. Secuenciador de entrega de producto. 
entrega del servicio. Este sincronismo se traduce en un compromiso de los elementos necesarios para el evento futuro. Los recursos necesarios típicamente son: Planta, Operador, Financieros, Tecnológicos, etc.

La entidad planta.- Es el recurso que determina la capacidad del servicio por evento. Por ejemplo para una Cita Médica la atención es personalizada, luego a un Ticket le corresponde un Paciente. Pero para el caso de un servicio de transporte la unidad de transporte (nave) se convierte en la planta, dependerá de su capacidad para la generación de lote de tickets. La generación de Tickets se considera como la fabricación de stock de Productos. Esta existencia de stock debe ser controlada por su Cuenta Control, sobre todo para la Venta y Entrega. Como se puede apreciar para el caso de los productos tipo servicios la Programación de estos, vía Eventos futuros constituyen existencias (stocks) listas para su venta. Figura 14. Todo Ticket es el resultado

\section{Tabla 1.}

$\begin{array}{ll}\text { SEC } & \text { OPERACIÓN } \\ 01 & \text { Check in de pasajero } \\ 02 & \text { Recepción Equipaje } \\ 03 & \text { Desarrollo del vuelo } \\ 04 & \text { Devolución de Equipaje }\end{array}$

de una Programación (evento creador) lo que significa determinar la ocurrencia de un hecho futuro donde se comprometen recursos, llámese recursos humanos, materiales, financieros y tecnológicos que desarrollarán el evento.

En todo sistema la Programación de Recursos para una Transacción, es en sí una Fabricación. Cuando un negocio oferta sus productos es porque espera que en este se den ventas futuras $y$, de acuerdo a esta estimación quedará determinada su Planta de Producción. De manera similar un empleado asiste a su jornada diaria porque así está Programado.

\section{Venta de Productos}

Consiste en definir los puntos del acuerdo del intercambio entre un cliente y la empresa (cierre de venta).

Solo se trata de un acuerdo no significa "entrega del producto" ni realizar pago alguno en el momento de la venta. La esencia de este tipo de transacción es la ASIGNACIÓN DE UN TICKET HACIA UN CLIENTE
(RESERVA DE STOCK). Así la transacción de Venta al consumarse genera un evento en el área comercial, generando cambios sobre las entidades que están involucradas como:

\begin{tabular}{|c|c|c|}
\hline Evento & $->$ & Crea el Hecho de Venta \\
\hline $\begin{array}{l}\text { Cliente } \\
\text { comprado }\end{array}$ & $->$ & Cambia Estadística de lo \\
\hline $\begin{array}{l}\text { Existencias } \\
\text { tickets. }\end{array}$ & $->$ & Cambia Stock Disponible de \\
\hline Cananl & $->$ & Cambia Estadísticas \\
\hline \multicolumn{3}{|c|}{ Ventas por Operador (Vendedor) y Planta. } \\
\hline Caja & $->$ & Actualiza Cuentas x Cobrar \\
\hline
\end{tabular}

ara el área generadora del Producto se dan cambios como el estado del Ticket de estado generado a estado asignado.

La situación de los recursos del ticket asignado queda comprometida de manera definitiva, porque mientras el ticket esté programado pero no asignado (vendido), podrían liberarse los recursos.

Con esta transacción de venta se logra fijar el compromiso, entre las partes:

- El negocio en entregar oportunamente el producto.

- El cliente en compensar al negocio por el producto.

Este compromiso se puede materializar en diferido o en el instante depende de:

- Plazo de entrega y

- Plazo de pago

Las ventas pueden tomar diferentes formas, por ejemplo:

Reserva de una Cita Médica, Vuelo, Función de Teatro, Matrícula Académica, Atención a Clientes entre otros. Para el ejemplo del Vuelo, se asignará un ticket al cliente que lo solicite y, este deberá aceptar la deuda generada por esta operación (resultado de la liquidación). Cerrada esta transacción, se procede a su liquidación del producto comprado, la cual debe seguir una Secuencia de Operaciones dada por el protocolo definido en la Etapa 1.

Para calcular el monto de la operación, se aplica la fórmula:

$$
\text { Monto }=\text { Cantidad } * \text { Precio }
$$

Esta operación se repite para cada existencia diferente que se venda. Tanto las cantidades como los precios pueden variar por Reglas de Negocio (RN) cuyo efecto impactará sobre su precio o cantidad final. 
Precio-fin = RN (Precio-catalogo)

Cantidad-fin $=$ RN (Cantidad-real)

Otras entidades como Cliente y Canal pueden igualmente estar sujetas a cumplir unas Reglas y Políticas de Negocio, por ejemplo: Tipo de cliente Vip o, Tipo de Canal virtual, igualmente se le aplicará un descuento.

\section{Vender Servicio $=$ Asignar un Ticket Ciclo: Entrega de productos}

En base al acuerdo definido en el ticket vendido se ejecuta o materializa lo pactado por parte de la empresa. Con la entrega del producto se finaliza el ciclo de vida. Lo recibido por el cliente debe dejarlo satisfecho. Sobre todo en el tema de servicios como atención médica, transporte, educación, etc.

La Etapa de Entrega del Servicio debe seguir estrictamente lo indicado por el protocolo. Se deben dar un conjunto de pasos $\mathrm{y}$, en cada uno validar las condiciones de acuerdo a las reglas y políticas de negocio. En cada uno de estos pasos se deben precisar: Objetivo, duración, resultado y costo. Figura 15.

Podemos observar las operaciones asociadas al vuelo comercial del ejemplo inicial. Se dan operaciones como: Registro de vuelo por parte del cliente (Check in), recepción de equipaje, desarrollo de vuelo y finalmente entrega de equipaje.

Se detalla la Entrega de un Vuelo Comercial:

Estas operaciones están secuenciadas según el protocolo de operaciones (definida en el ciclo I). En cada una de estas operaciones se aplican reglas y políticas de negocio. Por ejemplo el Check In tiene un tiempo límite, el equipaje no puede sobrepasar un peso límite. En esta etapa es realmente donde se opera el servicio, requiere de un ente que sincronice $u$ orqueste las diferentes operaciones que componen la entrega del servicio. Este sincronismo se plasma en el secuenciador o motor transaccional de operaciones. Este ente contiene el conocimiento para sincronizar y tomar decisiones en cada paso del protocolo, en forma automática, basado en parámetros.

En todo Evento de Entrega participan las Entidades del Negocio como:

- Evento del Servicio: Hecho

- Cliente: Recibe el Producto (Servicio)

- Existencia de Producto. Se atiende el ticket

- Operador desarrolla la Entrega el Producto
- Planta: Plataforma base para la entrega

- Caja: Descarga Cuenta pendiente.

Las macro transacciones se desarrollan en transacciones simples. Detallando al interior de la transacción encontramos operaciones que se desarrollan. Estas operaciones deben estar almacenadas en un repositorio de operaciones y se activan cuando nace el evento de la entrega. Cada operación igualmente se desarrollará mediante métodos siguiendo una secuencia lógica determinada. Es un motor operativo (Transaccional) encargado de secuenciar y ejecutar estas operaciones. El concepto de motor transaccional consiste en un conjunto de elemento manuales o automáticos capaces de ejecutar operaciones y métodos, siguiendo un protocolo de operaciones.

El Protocolo de entrega del servicio, debe ser definido y pactado con el cliente para luego ser respetado en el evento de entrega.

Cada operación del protocolo aplicará reglas y políticas de negocio, desarrollando el producto desde el inicio hasta su fin.

Respetando la secuencia lógica presentadas, todas estas operaciones de negocio existen $y$, se encuentran en el repositorio correspondiente esperando su activación o llamada al iniciarse el evento. El parámetro secuencia tiene rol de orquestador de operaciones. Basados en los componentes del repositorio, se definen el protocolo de entrega.

\section{CONCLUSIONES}

Se ha presentado el desarrollo (y la aplicación a través de un caso real) de un nuevo enfoque para plantear el Modelo Funcional de Negocio. Enfoque que facilitará el modelado de datos para la automatización del Sistema.

A diferencia de otros paradigmas, este método define de manera estándar solo cuatro Macro-Funciones válidas para todos los Sistemas.

Facilita y asegura la definición de Requerimientos vía determinación de Macro-Funciones y funciones detalladas, asegurando Robustez Semántica porque se revisa el ciclo de vida del Producto.

Esta revisión se aplica a modo de una lista de chequeo, permitiendo certificar un enfoque holístico del sistema que cubre los 360 grados al entorno del producto. 
Enfrentar la etapa de análisis basado en este enfoque estándar del Modelo de Negocio, proporciona claridad en los objetivos a lograr durante la etapa de estudio, basado en el propósito del sistema (Misión).

A nivel empresarial los Modelos Funcionales de Negocio existen en su estado estándar de manera universal, luego las definiciones de requerimientos del sistema van a consistir en personalizar vía parámetros sobre el patrón estándar, lo cual es una gran ventaja en tiempo, costo y calidad final del producto.

La definición de Requerimientos ahora consistirá en personalizar vía parámetros un esquema estándar del área de negocios en estudio.

\section{Sintetizando:}

1. Definir Misión del Área de Negocios.

2. Definir las cuatro Marco-funciones.

3. Plantear el modelo Paramétrico del sistema.

4. Contextualizar o personalizar el modelo a la realidad actual del usuario. Este proceso también conocido como adecuación o personalización paramétrica del modelo.

Es un proceso de configuración o programación paramétrica, que alinea el software a los objetivos y recursos con que cuenta el negocio en su contexto.

\section{REFERENCIAS}

[1] Klaus P, Sikora E The Co-Development of System Requirements and Functional Architecture

[2] Boehm B W Software Engineering Economics Prentice-Hall:New Jersey

[3] James M Sistemas de información

[4] James A and Stoner F Administración 5 ed

[5] Pressman R Ingeniería de Software

[6] Larman C UML y patrones

[7] Spyns P, Meersman R and Mustafa J Data modelling versus Ontology engineering

[8] Bueno E, Salvador M P, Reyes A 2012 Environmental sustainabilty from a stakeholder's perspective. A empirical analysis of the Spanish Hotel Industry International Journal of Production Research

[9] Bueno E 2007 Organización de Empresas:
Estructura, Procesos y Modelos Pirámide:Madrid.

[10] James M, James J Análisis y Diseño Orientado a Objetos Prentice Hall

[11] Hammer M, Champy J 1995 Reingeniería Norma:Colombia 7ed

[12] Chase R B, Aquilano N J and Jacobs R 2000 Administración de Producción y Operaciones. Manufactura y Servicios Mc. Graw Hill Irwin:Colombia, 8va ed

[13] Bueno E Organización de empresas: estructura, procesos y modelos

[14] Barceló M, Sánchez Schmitz G, Soltero A 2006 La Web Semántica como apoyo a la Gestión del Conocimiento y al Modelado Organizacional Revista vol 12, Ingeniería Informática:México

[15] Reyes S and Chalmeta R 2007 Modelado del Conocimiento Empresarial

[16] Colette R, Souveyet C and Ben Achour C 1998 Guiding Goal Modeling Using Scenarios IEEE Transactions on Software Engineering 24(12)

[17] Buschmann F and et al 1996 SAP POSA (PatternOriented Software Architecture)

[18] Buschmann F, Meunier R, Rohnert H, Sommerlad P and Stal M Pattern-Oriented Software Architecture: A System of Patterns vol 1 Wiley:India 